\title{
Adaptive Backstepping Sliding Mode Control of Trajectory Tracking for Robotic Manipulators
}

\author{
Zhu Dachang, ${ }^{1}$ Du Baolin $\left(\mathbb{D},{ }^{1}\right.$ Zhu Puchen $\mathbb{D}^{2},{ }^{2}$ and Wenqiang Wu ${ }^{1}{ }^{1}$ \\ ${ }^{1}$ School of Mechanical and Electrical Engineering, Guangzhou University, Guangzhou 510006, China \\ ${ }^{2}$ School of Automation, Guangdong University of Technology, Guangzhou 510006, China \\ Correspondence should be addressed to Wenqiang Wu; gz_wwq@gzhu.edu.cn
}

Received 31 May 2020; Revised 28 June 2020; Accepted 17 July 2020; Published 14 August 2020

Academic Editor: Shuping He

Copyright ( 2020 Zhu Dachang et al. This is an open access article distributed under the Creative Commons Attribution License, which permits unrestricted use, distribution, and reproduction in any medium, provided the original work is properly cited.

To achieve precise trajectory tracking of robotic manipulators in complex environment, the precise dynamic model, parameters identification, nonlinear characteristics, and disturbances are the factors that should be solved. Although parameters identification and adaptive estimate method were proposed for robotic control in many literature studies, the essential factors, such as coupling and friction, are rarely mentioned as it is difficult to build the precise dynamic model of the robotic manipulator. An adaptive backstepping sliding mode control is proposed to solve the precise trajectory tracking under external disturbances with complex environment, and the dynamic response characteristics of a two-link robotic manipulator are described in this paper. First, the Lagrange kinetic method is used to derive the precise dynamic model which includes the nonlinear factor with friction and coupling. Moreover, the dynamic model of two-link robotic manipulator is built. Second, the estimate function for the nonlinear part is selected, and backstepping algorithm is used for analyzing the stabilities of the sliding mode controller by using Lyapunov theory. Furthermore, the convergence of the proposed controller is verified subject to the external disturbance. At last, numerical simulation results are reported to demonstrate the effectiveness of the proposed method.

\section{Introduction}

Nowadays, with the development of modern industrial technology, robotic manipulators are widely used in automobile manufacturing, aerospace, electronic assembly, precision medical operation, and other fields. To obtain steady state accuracy and fast dynamical response, it is necessary for high precision of the trajectory tracking ability of robotic manipulator. Unfortunately, it is difficult to be satisfied on account of the nonlinear characteristics in complex environment like clearance of joints, friction, external disturbance, strong couple, and so on. The first stage of trajectory tracking is to establish the precise mathematical model of the robotic manipulator. However, the nonlinear part of the dynamic model of the robotic manipulator is ignored in many literatures [1-5] or parameter identification by many approaches [6-8]; even the torque in the joint space and the moment of inertia were ignored in [9]. By calculating kinetic energy, potential energy, and generalized force, the
Lagrange equation was utilized to build the dynamic equation for robotic manipulator $[10,11]$. As the recurrence relationship is not established between multiple links, it cannot be applied easily to the whole dynamic modeling for robotic manipulators. To overcome inaccuracy of the dynamical model of robotic manipulator, friction, clearance, and external disturbance were considered, and intelligent control strategies have been developed by many researchers for the uncertain manipulator [12-14], for example. In this paper, the dynamic equation with recurrence method by using Lagrange energy method is provided as an accurate mathematical model for precision trajectory tracking.

Furthermore, the design of intelligence controllers for nonlinear systems affected by disturbances is a topic that has been studied by several authors, and many different approaches have been proposed for this problem [15-17]. Backstepping is a systematic and recursive design method for nonlinear control applied to the feedback linearization system, which can guarantee global regulation and tracking 
performance $[18,19]$. It is a regression design method which combines the selection of Lyapunov function with the design of controller, and the virtual control is designed with the requirements step by step, and then, control law of complex uncertain system is finally designed. The basic design idea is to decompose the complex nonlinear system into subsystems of no more than systematic order and then design the Lyapunov function of each subsystem separately. On the basis of ensuring that the subsystem has guaranteed convergence, the control law of the subsystem is obtained. In the design of the next subsystem, the control law of the preceding subsystem is taken as the tracking target of this subsystem, and finally the control law of the last subsystem is obtained. By analogy, the control law of the entire closedloop system can be obtained, and the convergence of the closed-loop system is guaranteed by Lyapunov stability analysis method. Backstepping with intelligent algorithm can increase the quality of the transition process, reduce or even eliminate the uncertainty of the matching constraints, and provide a structured and systematic design method for the Lyapunov function design for complex nonlinear systems [20-23]. Sliding mode control (SMC) is another robust control method, which produces a switching control law (equivalent control law) to force the system to converge to the sliding surface within a boundary layer near the sliding surface under the convergence of the Lyapunov stability theory $[24,25]$. Trajectory tracking control of a 6-DOF pneumatically actuated Gough-Stewart parallel robot was investigated by Lafmejani [26], and position control of the pneumatic actuator was performed based on backstepping sliding mode controller according to the dynamic model of system. A methodology of dynamic analysis and control for a hybrid humanoid robot arm was presented in [27], and an adaptive backstepping sliding mode controller was developed for the parameters uncertainties and disturbances of the hybrid humanoid robot arm.

For the multi-input-multi-output (MIMO) nonlinear system with uncertainties and disturbances, sliding mode PI control with backstepping approach [28], presence of bounded uncertainties from unmodeled dynamic, parameters variations, disturbances, and visual fusion technology $[29,30]$, and asynchronous control with fuzzy approach [31] were proposed. To make the dynamic model of the robotic manipulator more accurate, an impedance-control strategy with dynamic compensation for interactive control of robot manipulators was presented in [32]. The kinematics and dynamics of multiple cooperative welding robot manipulators were studied on the basis of the Denavit-Hartenberg and Lagrange method, and adaptive neural control and dynamic movement primitives were considered in [33]. An adaptive backstepping sliding mode control of robotic manipulators is proposed in this paper to achieve the precision trajectory tracking with external disturbances. The main contributions of the current paper are summarized as follows:

(1) By using Lagrange energy function, the precise dynamic model of the robotic manipulator is built, and the nonlinear characteristics and uncertainties are analyzed. Furthermore, dynamic model of a two-link robotic manipulator is derived.

(2) According to the precision dynamic model of the two-link robotic manipulator, an estimate function of the nonlinear and coupling parts is proposed. Backstepping algorithm is used to construct the equivalent control law of sliding mode control through three steps, and the stability of the proposed controller is convergent by using Lyapunov theory.

This paper is organized in the following manner: Section 2 presents the precise dynamic modeling method and the precise dynamic model of the robotic manipulator. According to the previous method, the precise dynamic model of a two-link robotic manipulator is derived. Section 3 describes the sliding mode control with backstepping algorithm. Through the estimate function of nonlinear and coupling parts, the controller is proposed for the trajectory tracking under external disturbances, and its stability is discussed. Numerical simulation results are reported to demonstrate the effectiveness of the proposed method in Section 4. Finally, conclusions are provided in Section 5.

\section{Description of Robotic Manipulator and Dynamic Modeling}

To make the dynamic model recursive, the Newton-Euler method is used to establish all force balance between the links of the robotic manipulator, and the dynamic equation can be derived. Forward recursion is used for speed and acceleration transfer between all of the links and backward recursion is used for force transfer from the end-effector to each link of the robotic manipulator.

\subsection{Description of Link Parameters of Robotic Manipulator.} The dynamic parameters which describe the dynamic model are important for the control algorithms, effective simulation results, and accurate trajectory tracking algorithms. Dynamic equation of the robotic manipulator with $n$-DOF has been characterized in many literature studies [1-11] as follows:

$$
D(q) \ddot{q}+C(q, \dot{q}) \dot{q}+G(q)+F(q, \dot{q})+\tau_{d}=\tau,
$$

where $q, \dot{q}, \ddot{q} \in R^{n}$ are the link position, velocity, and acceleration vectors, respectively, $D(q) \in R^{n \times n}$ is the symmetric positive definite inertia matrix, $C(q, \dot{q}) \in R^{n \times n}$ is the Coriolis or centrifugal forces, $G(q) \in R^{n \times 1}$ consolidates the gravitational force, $F(q, \dot{q}) \in R^{n \times 1}$ incorporates the friction terms, and $\tau_{d}$ represents external disturbance.

However, quadratic velocity terms and dynamic coupling terms are not taken into account. So, the problem of model accuracy cannot be solved essentially only through parameter identification and compensation methods. The kinematic description of the $i^{\text {th }}$ link is shown in Figure 1.

An infinitesimal element $e_{i}$ is selected which lies on the $i^{\text {th }}$ link, and the position of the center of mass $c_{i}$ is represented by vector $p_{i}$, the position vector of $e_{i}$ is presented by vector $p_{i}^{*}$, and the position vector from the center of mass to 


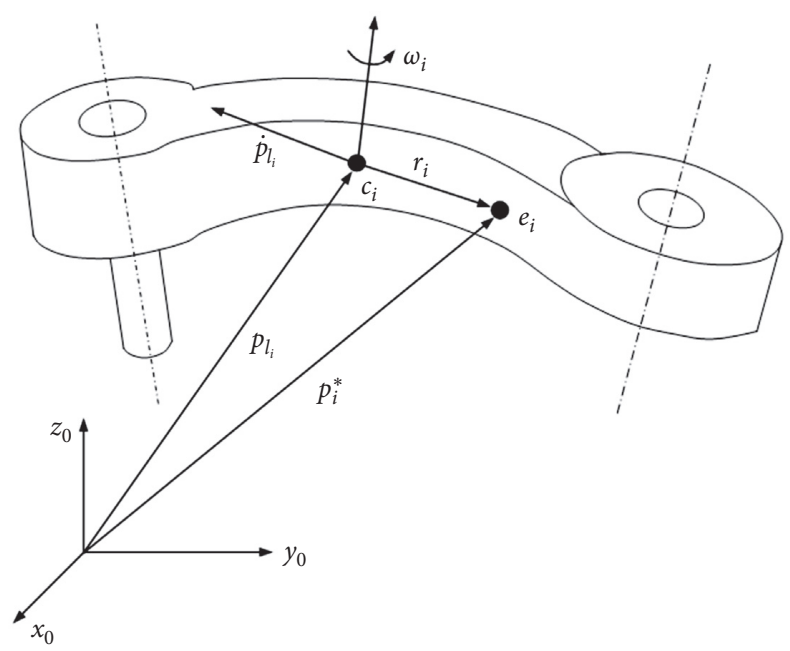

Figure 1: Kinematic description of the $i^{\text {th }}$ link.

the infinitesimal element is $r_{i}$. When integrating in the whole area of the $i^{\text {th }}$ link, the infinitesimal element can represent the motion performance of the whole link.

The kinetic energy component $E_{k l_{i}}$ of the $i^{\text {th }}$ link can be given by

$$
E_{k l_{i}}=\frac{1}{2} \int_{V_{l_{i}}}\left(\dot{p}_{i}^{*}\right)^{T} \dot{p}_{i}^{*} \rho \mathrm{d} V,
$$

where $\dot{p}_{i}^{*}$ is the linear velocity vector of the infinitesimal element, $\rho$ is material density, and $\mathrm{d} V$ is the volume of the infinitesimal element.

The position vector relationship between $c_{i}$ and $e_{i}$ is satisfied with

$$
r_{i}=\left[\begin{array}{lll}
r_{i x} & r_{i y} & r_{i z}
\end{array}\right]^{T}=p_{i}^{*}-p_{l_{i}},
$$

where $p_{l_{i}}$ is calculated by centroid theorem as

$$
p_{l_{i}}=\frac{1}{m_{l_{i}}} \int_{V_{l_{i}}} p_{i}^{*} \rho \mathrm{d} V
$$

where $m_{l,}$ is the mass of the $i^{\text {th }}$ link.

Differentiating equation (3) with respect to time, one can get

$$
\dot{p}_{i}^{*}=\dot{p}_{l_{i}}+\omega_{i} \times r_{i}=\dot{p}_{l_{i}}+S\left(\omega_{i}\right) r_{i},
$$

where $\dot{p}_{l_{i}}$ is the linear velocity vector of the center of mass, $\omega_{i}$ is angular velocity of the infinitesimal element around the center of mass, and $S\left(\omega_{i}\right)$ is the antisymmetric matrix of three-dimensional vector $\omega_{i}=\left[\begin{array}{lll}\omega_{i x} & \omega_{i y} & \omega_{i z}\end{array}\right]^{T}$ and defined as follows:

$$
S\left(\omega_{i}\right)=\left[\begin{array}{ccc}
0 & -\omega_{i z} & \omega_{i y} \\
\omega_{i z} & 0 & -\omega_{i x} \\
-\omega_{i y} & \omega_{i x} & 0
\end{array}\right] .
$$

Substituting equation (5) into equation (2), one can obtain

$$
E_{k l_{i}}=\frac{1}{2} \int_{V_{l_{i}}}\left(\dot{p}_{l_{i}}+S\left(\omega_{i}\right) r_{i}\right)^{T}\left(\dot{p}_{l_{i}}+S\left(\omega_{i}\right) r_{i}\right) \rho \mathrm{d} V .
$$

Equation (7) shows that the kinetic energy of each link consists of three parts, described as follows:

(1) Translational kinetic energy is expressed as

$$
\frac{1}{2} \int_{V_{l_{i}}} \dot{p}_{l_{i}}^{T} \dot{p}_{l_{i}} \rho \mathrm{d} V=\frac{1}{2} m_{l_{i}} \dot{p}_{l_{i}}^{T} \dot{p}_{l_{i}}
$$

(2) Implicated motion kinetic energy is expressed as

$$
2\left(\frac{1}{2} \int_{V_{l_{i}}} \dot{p}_{l_{i}} S\left(\omega_{i}\right) r_{i} \rho \mathrm{d} V\right)=2\left(\frac{1}{2} \dot{p}_{l_{i}}^{T} S\left(\omega_{i}\right)\right) \int_{V_{l_{i}}}\left(p_{i}^{*}-p_{l_{i}}\right) \rho \mathrm{d} V=0 .
$$

(3) Rotation kinetic energy, combining with equation (6), is expressed as

$$
\begin{aligned}
\frac{1}{2} \int_{V_{l_{i}}} r_{i}^{T} S^{T}\left(\omega_{i}\right) S\left(\omega_{i}\right) r_{i} \rho \mathrm{d} V & =\frac{1}{2} \omega_{i}^{T}\left(\int_{V_{l_{i}}} S^{T}\left(r_{i}\right) S\left(r_{i}\right) \rho \mathrm{d} V\right) \omega_{i} \\
& =\frac{1}{2} \omega_{i}^{T} I_{l_{i}} \omega_{i},
\end{aligned}
$$

where $S\left(\omega_{i}\right) r_{i}=-S\left(r_{i}\right) \omega_{i} ; I_{l_{i}}$ is defined as

$I_{l_{i}}=\left[\begin{array}{ccc}\int\left(r_{i y}^{2}+r_{i z}^{2}\right) \rho \mathrm{d} V & -\int r_{i x} r_{i y} \rho \mathrm{d} V & -\int r_{i x} r_{i z} \rho \mathrm{d} V \\ -\int r_{i x} r_{i y} \rho \mathrm{d} V & \int\left(r_{i x}^{2}+r_{i z}^{2}\right) \rho \mathrm{d} V & -\int r_{i z} r_{i y} \rho \mathrm{d} V \\ -\int r_{i x} r_{i z} \rho \mathrm{d} V & -\int r_{i z} r_{i y} \rho \mathrm{d} V & \int\left(r_{i y}^{2}+r_{i x}^{2}\right) \rho \mathrm{d} V\end{array}\right]$, 
(1) where $I_{l}$ is symmetric matrix and denotes inertia tensor related to the center of mass of the $i^{\text {th }}$ link in base coordinate system.

Combining equation (8) with equation (10), the kinetic energy of the $i^{\text {th }}$ link can be given by

$$
E_{k l_{i}}=\frac{1}{2} m_{l_{i}} \dot{q}^{T}\left(J_{p}^{\left(l_{i}\right)}\right)^{T} J_{p}^{\left(l_{i}\right)} \dot{q}+\frac{1}{2} \dot{q}^{T}\left(J_{o}^{\left(l_{i}\right)}\right)^{T} I_{l_{i}} J_{o}^{\left(l_{i}\right)} \dot{q}
$$

where $q=\left(\begin{array}{llll}q_{1} & q_{2} & \cdots & q_{i}\end{array}\right)^{T}$ is joint variable vector.

The kinetic energy component of the motor of $i^{\#}$ joint can be calculated in a similar way. Under the assumption of rigid transmission, there is

$$
k_{t i} \dot{q}_{i}=\dot{\theta}_{m_{i}}
$$

where $k_{t i}$ is the transmission ratio of gear speed reducer and $\theta_{m_{i}}$ is the rotor angular position.

With the law of angular velocity synthesis, the total angular velocity is derived as follows:

$$
\omega_{m_{i}}=\omega_{i-1}+k_{t i} \dot{q}_{i} z_{m_{i}}
$$

where $\omega_{i-1}$ is the angular velocity of the $(i-1)^{\#}$ link and $z_{m_{i}}$ is the unit vector of rotor axis.

The linear and angular velocities of the rotor center of mass can be expressed as

$$
\begin{aligned}
& \dot{p}_{m_{i}}=J_{p}^{\left(m_{i}\right)} \dot{q}, \\
& \omega_{m_{i}}=J_{o}^{\left(m_{i}\right)} \dot{q},
\end{aligned}
$$

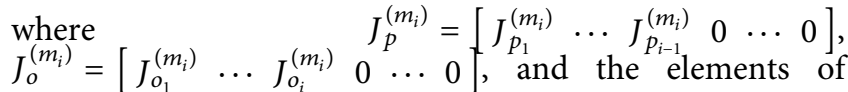
matrix are given by

$$
\begin{aligned}
& J_{p_{i}}^{\left(m_{i}\right)}=z_{i-1} \times\left(p_{m_{i}}-p_{i-1}\right), \\
& J_{o_{j}}^{\left(m_{i}\right)}= \begin{cases}J_{o_{j}}^{\left(l_{i}\right)}, & j=1,2, \ldots, i-1, \\
k_{t i} z_{m_{i}}, & j=i .\end{cases}
\end{aligned}
$$

If the rotor turns around its center, then, $J_{p_{i}}^{\left(m_{i}\right)}=0$.

So, the kinetic energy component $E_{k m_{i}}$ of the $i^{\text {th }}$ rotor can be given by

$$
E_{k m_{i}}=\frac{1}{2} m_{m_{i}} \dot{q}^{T}\left(J_{p}^{\left(m_{i}\right)}\right)^{T} J_{p}^{\left(m_{i}\right)} \dot{q}+\frac{1}{2} \dot{q}^{T}\left(J_{o}^{\left(m_{i}\right)}\right)^{T} I_{m_{i}} J_{o}^{\left(m_{i}\right)} \dot{q}
$$

where $m_{m_{i}}$ is the mass of the rotor and $I_{m_{i}}$ is the inertia matrix of rotor.

Summing different components of a single link in (12) and a single motor in (17), the total kinetic energy of the robotic manipulator can be calculated and yields

$$
E_{k}=E_{k l_{i}}+E_{k m_{i}}=\frac{1}{2} \sum_{i=1}^{n} \sum_{j=1}^{n} b_{i j}(q) \dot{q}_{i} \dot{q}_{j}=\frac{1}{2} \dot{q}^{T} B(q) \dot{q}
$$

where $B(q)$ is the inertia matrix, satisfied with

$$
B(q)=\sum_{i=1}^{n}\left(m_{l_{i}}\left(J_{p}^{\left(l_{i}\right)}\right)^{T} J_{p}^{\left(l_{i}\right)}+\left(J_{o}^{\left(l_{i}\right)}\right)^{T} I_{l_{i}} J_{o}^{\left(l_{i}\right)}+m_{m_{i}}\left(J_{p}^{\left(m_{i}\right)}\right)^{T} J_{p}^{\left(m_{i}\right)}+\left(J_{o}^{\left(m_{i}\right)}\right)^{T} I_{m_{i}} J_{o}^{\left(m_{i}\right)}\right)
$$
follows:

The potential energy of the $i^{\text {th }}$ link can be calculated as

$$
U_{l_{i}}=-\int_{V_{l_{i}}} g_{0}^{T} p_{i}^{*} \rho \mathrm{d} V=-m_{l_{i}} g_{0}^{T} p_{l_{i}}
$$

where $g_{0}$ is acceleration vector of gravity in the base coordinate system.

So, the total potential energy $U$ of the robotic manipulator can be expressed as

$$
U=\sum_{i=1}^{n}\left(U_{l_{i}}+U_{m_{i}}\right)=-\sum_{i=1}^{n}\left(m_{l_{i}} g_{0}^{T} p_{l_{i}}+m_{m_{i}} g_{0}^{T} p_{m_{i}}\right)
$$

where $p_{m_{i}}$ is the position vector of rotor center of mass.

According to (18) and (21), the Lagrange function is constructed as follows:

$$
L(q, \dot{q})=E_{k}(q, \dot{q})-U(q)
$$

The dynamic equation is derived by using Lagrange function, yielding

$$
\tau_{i}=\frac{\mathrm{d}}{\mathrm{d} t}\left(\frac{\partial L}{\partial \dot{q}_{i}}\right)-\frac{\partial U}{\partial q_{i}}
$$

where

$$
\begin{aligned}
\frac{\mathrm{d}}{\mathrm{d} t}\left(\frac{\partial L}{\partial \dot{q}_{i}}\right) & =\frac{\mathrm{d}}{\mathrm{d} t}\left(\frac{\partial E_{k}}{\partial \dot{q}_{i}}\right)=\sum_{j=1}^{n} b_{i j}(q) \ddot{q}_{j}+\sum_{j=1}^{n} \sum_{k=1}^{n} \frac{\partial b_{i j}(q)}{\partial q_{k}} \dot{q}_{k} \dot{q}_{j}, \\
\frac{\partial E_{k}}{\partial q_{i}} & =\frac{1}{2} \sum_{j=1}^{n} \sum_{k=1}^{n} \frac{\partial b_{i j}(q)}{\partial q_{i}} \dot{q}_{k} \dot{q}_{j}, \\
\frac{\partial U}{\partial q_{i}} & =-\sum_{j=1}^{n}\left(m_{l_{j}} g_{0}^{T} J_{p_{i}}^{\left(l_{i}\right)}(q)+m_{m_{j}} g_{0}^{T} J_{p_{i}}^{\left(m_{i}\right)}(q)\right)=g_{i}(q) .
\end{aligned}
$$

The dynamic equation of robotic manipulator with $n$-DOF can be derived as

$$
\sum_{j=1}^{n} b_{i j}(q) \ddot{q}_{j}+\sum_{j=1}^{n} \sum_{k=1}^{n} h_{i j k}(q) \dot{q}_{k} \dot{q}_{j}+g_{i}(q)=\tau_{i}, \quad i=1,2, \ldots, n,
$$


where $h_{i j k}=\left(\partial \dot{b}_{i j} / \partial q_{k}\right)-\left((1 / 2)\left(\partial b_{i j} / \partial q_{i}\right)\right)$.

Comparing (25) with (1), the elements $c_{i j}$ of $C(q, \dot{q})$ should be satisfied with

$$
\sum_{j=1}^{n} c_{i j} q_{j}=\sum_{j=1}^{n} \sum_{k=1}^{n} h_{i j k} \dot{q}_{k} \dot{q}_{j}
$$

Considering the viscous friction and Coulomb friction, equation (25) can also be rewritten as follows:

$$
B(q) \ddot{q}+C(q, \dot{q}) \dot{q}+F_{v} \dot{q}+F_{s} \operatorname{sgn}(\dot{q})+g(q)=\tau-J^{T}(q) h_{e},
$$

where $h_{e}$ is contact force of end-effector, $J(q)$ is velocity Jacobi matrix, $F_{v} \dot{q}$ is the torque of viscous friction, and $F_{s} \operatorname{sgn}(\dot{q})$ is the torque of Coulomb friction.

2.2. Parameters and Dynamic Model of Two-Link Robotic Manipulator. The dynamic mathematical model for a rigid planar robotic manipulator having two links and a contact surface with the external force acting on the surface is shown in Figure 2. According to the coordinate system $\{o-x y\}$, it consists of two links having link lengths $l_{1}$ and $l_{2}$ with their centers of mass $m_{1}$ and $m_{2}$ lying at the middle of links, respectively. The length of the center of mass is $p_{1}$ and $p_{2}$, respectively.

The Lagrange method is used to build the precise dynamic model of a two-link robotic manipulator with their nominal values as listed in Table 1 .

The total kinetic energy of the two-link robotic manipulator is

$$
\begin{aligned}
E_{k}= & E_{k 1}+E_{k 2}=\frac{1}{2} m_{1} p_{1}^{2} \dot{\theta}_{1}^{2}+\frac{1}{2} m_{2} l_{1}^{2} \dot{\theta}_{1}^{2}+\frac{1}{2} m_{2} p_{2}^{2}\left(\dot{\theta}_{1}+\dot{\theta}_{2}\right)^{2} \\
& +m_{2} l_{1} p_{2}\left(\dot{\theta}_{1}^{2}+\dot{\theta}_{1} \dot{\theta}_{2}\right) \cos \theta_{2},
\end{aligned}
$$

where $\theta_{1}$ and $\theta_{2}$ are joint variables of the two-link robotic manipulator.

The total potential energy of the two-link robotic manipulator is

$$
E_{p}=E_{p 1}+E_{p 2}=m_{1} g p_{1} \cos \theta_{1}+m_{2} g\left(l_{1} \cos \theta_{1}+p_{2} \cos \left(\theta_{1}+\theta_{2}\right)\right) \text {. }
$$

By using (23), the dynamic equation of two-link robotic manipulator can be derived as

$$
\tau_{1}=\frac{\mathrm{d}}{\mathrm{d} t} \frac{\partial L}{\partial \dot{\theta}_{1}}-\frac{\partial L}{\partial \theta_{1}}=D_{11} \ddot{\theta}_{1}+D_{12} \ddot{\theta}_{2}+D_{112} \dot{\theta}_{1} \dot{\theta}_{2}+D_{122} \dot{\theta}_{2}^{2}+D_{1},
$$

where $s_{1}=\sin \theta_{1}, c_{1}=\cos \theta_{1} s_{12}=\sin \left(\theta_{1}+\theta_{2}\right)$, and

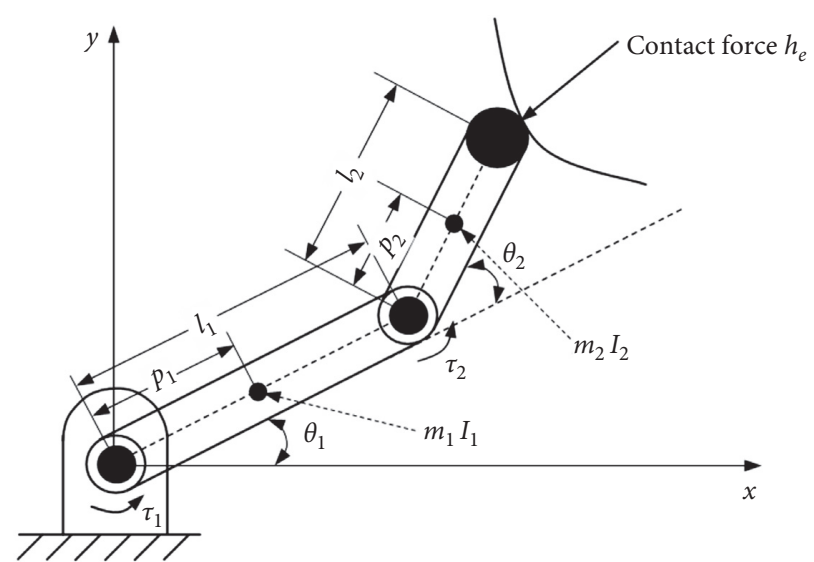

FIGURE 2: Two-link robotic manipulator plant with contact force at tip.

TABLE 1: Variables description of the two-link robotic manipulator.

Description

Nominal value

Center of mass link 1: $m_{1}(\mathrm{~kg})$

Length of link 1: $l_{1}(\mathrm{~m})$

Length of center of mass link 1: $p_{1}(\mathrm{~m})$

Centroid inertia of link 1: $I_{1}\left(\mathrm{kgm}^{2}\right)$

Center of mass link 2: $m_{2}(\mathrm{~kg})$

Length of link 2: $l_{2}(\mathrm{~m})$

Length of center of mass link 2: $p_{2}(\mathrm{~m})$

Centroid inertia of link 2: $I_{2}\left(\mathrm{kgm}^{2}\right)$

$$
\left\{\begin{array}{l}
D_{11}=m_{1} p_{1}^{2}+m_{2} p_{2}^{2}+m_{2} l_{1}^{2}+2 m_{2} l_{1} p_{2} c_{2}, \\
D_{12}=m_{2} p_{2}^{2}+m_{2} l_{1} p_{2} c_{2}, \\
D_{112}=-2 m_{2} l_{1} p_{2} s_{2}, \\
D_{122}=-m_{2} l_{1} p_{2} s_{2} \\
D_{1}=\left(m_{1} p_{1}+m_{2} l_{1}\right) g s_{1}+m_{2} p_{2} g s_{12} .
\end{array}\right.
$$

Meanwhile, the torque of joint $2 \tau_{2}$ can be also derived as $\tau_{2}=\frac{\mathrm{d}}{\mathrm{d} t} \frac{\partial L}{\partial \dot{\theta}_{2}}-\frac{\partial L}{\partial \theta_{2}}=D_{21} \ddot{\theta}_{1}+D_{22} \ddot{\theta}_{2}+D_{212} \dot{\theta}_{1} \dot{\theta}_{2}+D_{211} \dot{\theta}_{2}^{2}+D_{2}$,

where

$$
\left\{\begin{array}{l}
D_{21}=m_{1} p_{2}^{2}+m_{2} l_{1} p_{2} c_{2}, \\
D_{22}=m_{2} p_{2}^{2} \\
D_{212}=0 \\
D_{211}=m_{2} l_{1} p_{2} s_{2} \\
D_{2}=m_{2} p_{2} g s_{12}
\end{array}\right.
$$

Combining (30) with (32), the robotic plant can be rewritten with following mathematical model:

$$
\begin{aligned}
& {\left[\begin{array}{ll}
D_{11} & D_{12} \\
D_{21} & D_{22}
\end{array}\right]\left[\begin{array}{l}
\ddot{\theta}_{1} \\
\ddot{\theta}_{2}
\end{array}\right]+\left[\begin{array}{l}
D_{112} \\
D_{212}
\end{array}\right]\left[\begin{array}{l}
\dot{\theta}_{1} \dot{\theta}_{2} \\
\dot{\theta}_{2} \dot{\theta}_{1}
\end{array}\right]+\left[\begin{array}{l}
D_{211} \\
D_{122}
\end{array}\right]\left[\begin{array}{c}
\dot{\theta}_{2}^{2} \\
\dot{\theta}_{1}^{2}
\end{array}\right]} \\
& +\left[\begin{array}{l}
D_{1} \\
D_{2}
\end{array}\right]=\left[\begin{array}{l}
\tau_{1} \\
\tau_{2}
\end{array}\right] .
\end{aligned}
$$


Equation (14) completely represents the relationship between actuated torque and displacement, velocity, and acceleration in the joint space. The issues with $D_{11}$ and $D_{22}$ represent the moment of inertia caused by the acceleration of joint 1 and joint 2, respectively. The issues with $D_{12}$ and $D_{21}$ represent the moment of inertia of the acceleration coupling between two joints. The issues with $D_{122}$ and $D_{211}$ represent the coupling moment term of the centripetal force caused by the velocity between two joints. The issues with $D_{112}$ and $D_{212}$ represent the coupling moment term of the Coriolis force between two joints. $D_{1}$ and $D_{2}$ represent the gravity moment term. Considering the effect of centroid inertia, (35) can be modified as

$$
\begin{aligned}
& D_{11}^{\prime}=I_{1}+I_{2}+D_{11}, \\
& D_{12}^{\prime}=I_{2}+D_{12}, \\
& D_{21}^{\prime}=I_{2}+D_{21}, \\
& D_{22}^{\prime}=I_{2}+D_{22} .
\end{aligned}
$$

Furthermore, to consider the effect of the contact force acting on the end-effector, the right side of (34) can be replaced by

$$
\tau^{\prime}=\tau-J^{T}(q) h_{e}
$$

where $h_{e}$ is the force and moment vector applied by the endeffector in the working environment and $J^{T}(q)$ is the velocity Jacobian matrix, yielding

$$
J(q)=\left[\begin{array}{cc}
-l_{1} s_{1}-l_{2} s_{12} & -l_{2} s_{12} \\
l_{1} c_{1}+l_{2} c_{12} & l_{2} c_{12}
\end{array}\right] .
$$

\section{Adaptive Backstepping Sliding Mode Control Algorithm}

In this section, an adaptive backstepping sliding mode controller is presented which achieves precision trajectory tracking property by guaranteeing the robustness and stability of the closed-loop system of robotic manipulator. The uncertainties included in the system are required for compensating the external disturbances and nonlinear dynamics in terms described as (27). The adaptive backstepping sliding mode control is constructed at the final step.

The inverse dynamic equation can be expressed as

$$
\begin{aligned}
\ddot{q}= & -B^{-1}(q)\left[C(q, \dot{q}) \dot{q}+F_{v} \dot{q}+F_{s} \operatorname{sgn}(\dot{q})+g(q)\right] \\
& +B^{-1}(q)\left(\tau-J^{T}(q) h_{e}\right) .
\end{aligned}
$$

Property 1. $B(q)$ is the $n \times n$ symmetric inertial matrix and bounded:

$$
c_{1}\|A\|^{2} \leq A^{T} B(q) A \leq c_{2}\|A\|^{2}, \quad \forall A \in R^{n \times 1},
$$

where $c_{1}$ and $c_{2}$ are positive constants.

Property 2. $\dot{B}(q)-2 C(q, \dot{q})$ is skew symmetric matrix and satisfies

$$
A^{T}(\dot{B}(q)-2 C(q, \dot{q})) A=0
$$

Property 3. $C(q, \dot{q}), g(q)$, and $F_{v} \dot{q}+F_{s} \operatorname{sgn}(\dot{q})$ are bounded as follows:

$$
\begin{aligned}
\|C(q, \dot{q}) \dot{q}\| & \leq c_{k}\|\dot{q}\|^{2}, \\
\|g(q)\| & \leq g_{k}, \\
\left\|F_{v} \dot{q}+F_{s} \operatorname{sgn}(\dot{q})\right\| & \leq f_{k}\|\dot{q}\|,
\end{aligned}
$$

where $c_{k}, g_{k}$, and $f_{k}$ are positive constants.

Property 4. $J^{T}(q) h_{e} \in R^{n}$ is unknown disturbance and bounded as

$$
\left\|J^{T}(q) h_{e}\right\| \leq \tau_{k}, \quad \tau_{k}>0 .
$$

Defining vectors $x_{1}=q, x_{2}=\dot{q}, x=\left[\begin{array}{ll}x_{1} & x_{2}\end{array}\right]^{T}$, the desired track is defined as $x_{d}=\left[\begin{array}{ll}x_{1 d} & x_{2 d}\end{array}\right]^{T}$, so the state equation of the robotic manipulator can be given by

$$
\begin{aligned}
& \dot{x}_{1}=x_{2}, \\
& \dot{x}_{2}=B^{-1}(q)(\tau-C(q, \dot{q}) \dot{q}-g(q)-\varphi(q, \dot{q})),
\end{aligned}
$$

where $\varphi(q, \dot{q})$ is defined as the disturbance nonlinear function.

Assuming that $q_{d}$ is the desired trajectory of the robotic manipulator in joint space, select $e_{1}$ as the trajectory tracking error; it is defined as

$$
e_{1}=q-q_{d}=x_{1}-x_{1 d} .
$$

Differentiating (44) with respect to time, one can get

$$
\dot{e}_{1}=\dot{q}-\dot{q}_{d}=\dot{x}_{1}-\dot{x}_{1 d}=x_{2}-\dot{x}_{1 d}
$$

By using backstepping algorithm, let $e_{2}$ be a virtual input; then, the feedback control law can be expressed as

$$
e_{2}=\dot{e}_{1}+k_{1} e_{1}
$$

where $k_{1} \in R^{n \times n}$ is a positive definite diagonal coefficient matrix according to the DOF of robotic manipulator.

Theorem 1. Consider a robotic manipulator with n-DOF with the dynamic in (28), by designing the following sliding mode controller as

$$
\tau_{e q}=-h\left[\lambda\left(e_{2}-k_{1} e_{1}\right)+B^{-1}(q)(\tau-C(q, \dot{q}) \dot{q}-g(q)-\varphi(q, \dot{q}))-\ddot{q}_{d}+k_{1} \dot{e}_{1}\right]=-h s
$$




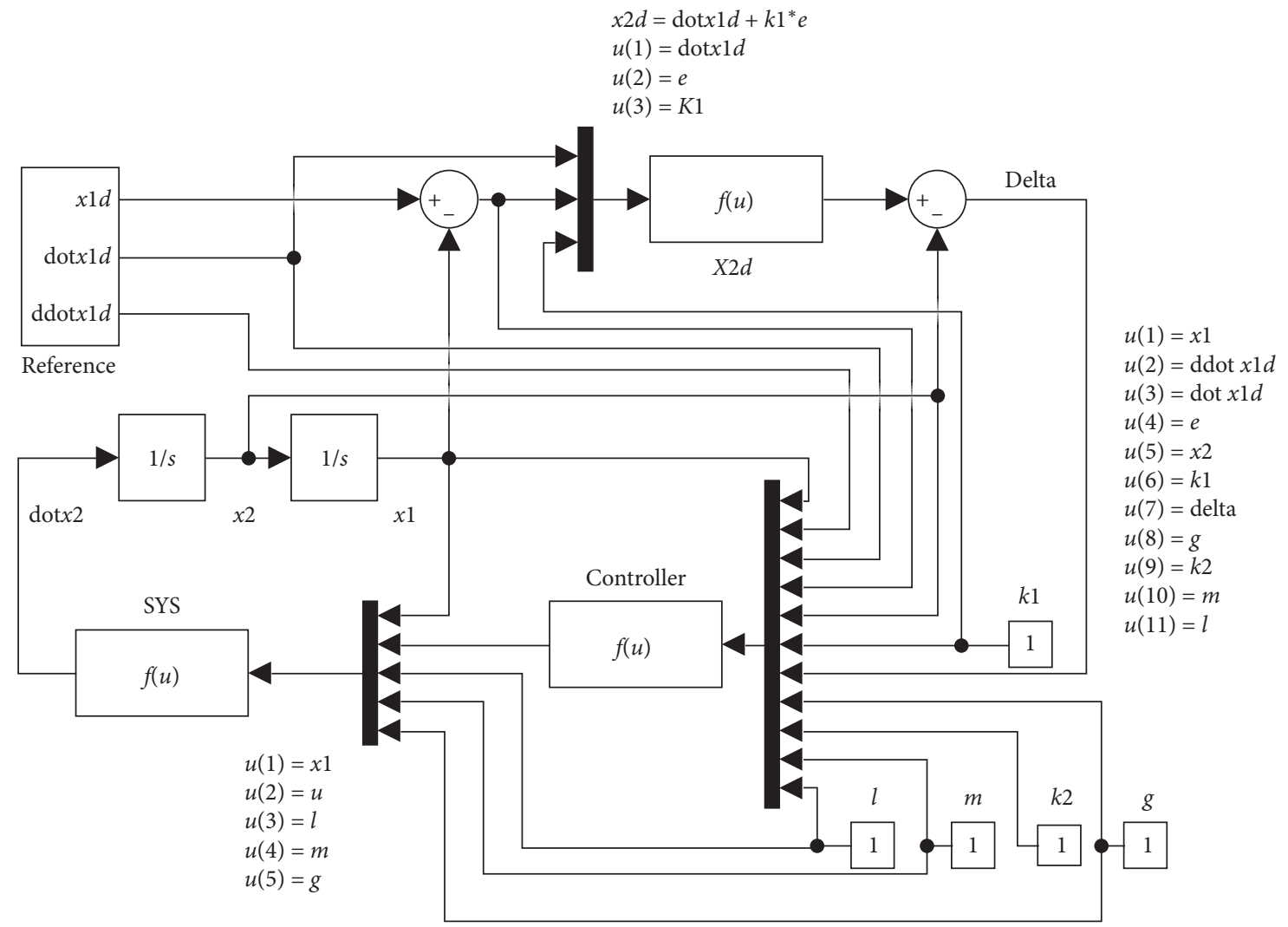

FIGURE 3: Schematic for backstepping algorithm by using Matlab ${ }^{@}$ software.

where $h \in R^{n \times n}$ is a positive definite diagonal coefficient matrix.

Select the Lyapunov function of the first step as

$$
V_{1}=\frac{1}{2} e_{1}^{T} e_{1} .
$$

Differentiating (48) with respect to time, one can get

$$
\dot{V}_{1}=e_{1}^{T} \dot{e}_{1}=e_{1}^{T}\left(e_{2}-k_{1} e_{1}\right)=e_{1}^{T} e_{2}-e_{1}^{T} k_{1} e_{1} .
$$

If $e_{2}=0$, then $\dot{V}_{1}=-e_{1}^{T} k_{1} e_{1} \leq 0$. So, the sliding surface function is defined as $s=\lambda e_{1}+e_{2}$, and the Lyapunov function of the second step is selected as

$$
V_{2}=V_{1}+\frac{1}{2} s^{T} s
$$

where $\lambda \in R^{n \times n}$ is a positive definite diagonal coefficient matrix according to the DOF of robotic manipulator.

Differentiating (50) with respect to time, one can get

$$
\begin{aligned}
\dot{V}_{2}= & e_{1}^{T} e_{2}-e_{1}^{T} k_{1} e_{1}+s^{T}\left[\lambda \dot{e}_{1}+\dot{e}_{2}\right] \\
= & e_{1}^{T} e_{2}-e_{1}^{T} k_{1} e_{1}+s^{T}\left[\lambda\left(e_{2}-k_{1} e_{1}\right)+\ddot{e}_{1}+k_{1} \dot{e}_{1}\right] \\
= & e_{1}^{T} e_{2}-e_{1}^{T} k_{1} e_{1}+s^{T}\left[\lambda\left(e_{2}-k_{1} e_{1}\right)+B^{-1}(q)(\tau-C(q, \dot{q}) \dot{q}\right. \\
& \left.-g(q)-\varphi(q, \dot{q}))-\ddot{q}_{d}+k_{1} \dot{e}_{1}\right] .
\end{aligned}
$$

An adaptive algorithm can be assigned for nonlinear function $\varphi(q, \dot{q})$, yielding

$$
\begin{aligned}
& e_{\varphi(q, \dot{q})}=\varphi(q, \dot{q})-\widehat{\varphi}(q, \dot{q}), \\
& \dot{e}_{\varphi(q, \dot{q})}=-\dot{\widehat{\varphi}}(q, \dot{q}),
\end{aligned}
$$

where $e_{\varphi(q, \dot{q})}$ is the estimation error and $\widehat{\varphi}(q, \dot{q})$ is the estimated value of the nonlinear function $\varphi(q, \dot{q})$.

The Lyapunov function of the third step is selected as

$$
V_{3}=V_{2}+\frac{1}{2} k_{2} e_{\varphi(q, q)}^{T} e_{\varphi(q, q)} .
$$

Differentiating (53) with respect to time, one can get

$$
\dot{V}_{3}=\dot{V}_{2}-k_{2} e_{\varphi(q, \dot{q})}^{T}\left(\dot{\hat{\varphi}}(q, \dot{q})+\frac{1}{k_{2}}\left(\left(M^{-1}(q)\right)^{T} s\right)\right) \text {. }
$$

So, the adaptive control law is derived as $\dot{\widehat{\varphi}}(q, \dot{q})=-1 / k_{2}\left(\left(M^{-1}(q)\right)^{T} s\right)$.

Considering the boundary of $\varphi(q, \dot{q})$ and Property 3, (54) can be rewritten as follows:

$$
\begin{aligned}
\dot{V}_{3} & \leq e_{1}^{T} e_{2}-e_{1}^{T} k_{1} e_{1}-\left(\lambda e_{1}+e_{2}\right)^{T} h\left(\lambda e_{1}+e_{2}\right) \\
& =-\left[\begin{array}{ll}
e_{1} & e_{2}
\end{array}\right]^{T} M\left[\begin{array}{ll}
e_{1} & e_{2}
\end{array}\right],
\end{aligned}
$$

where 


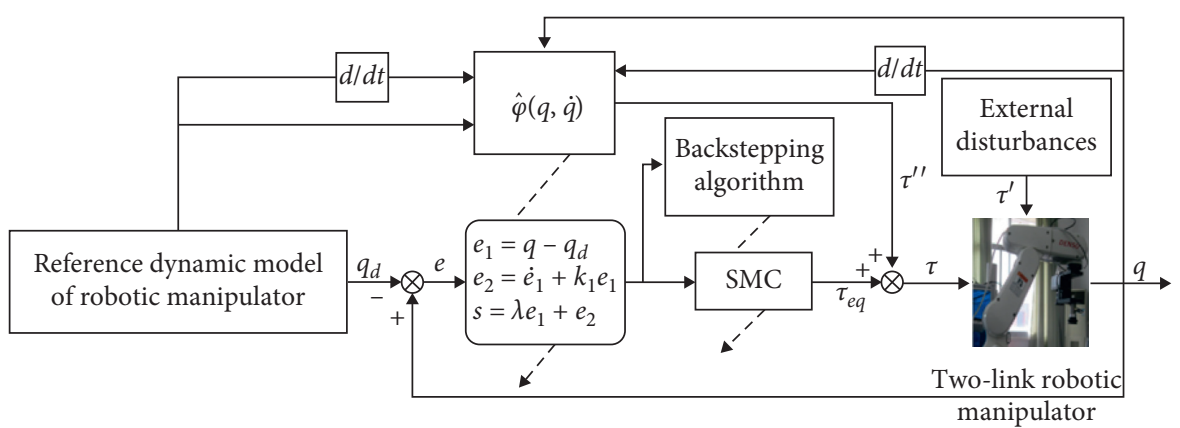

FIGURE 4: Schematic of the proposed control strategy.

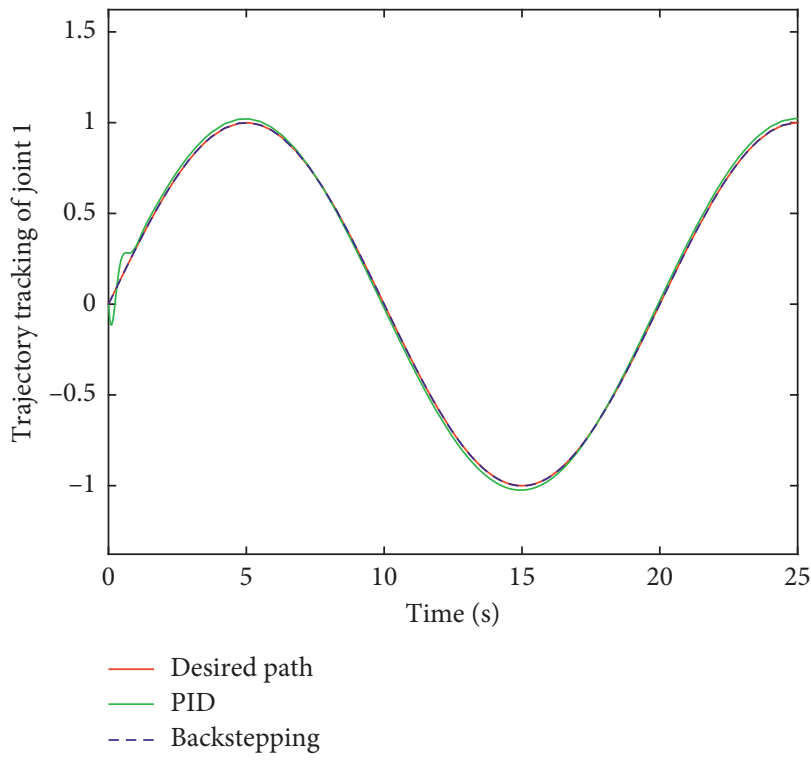

(a)

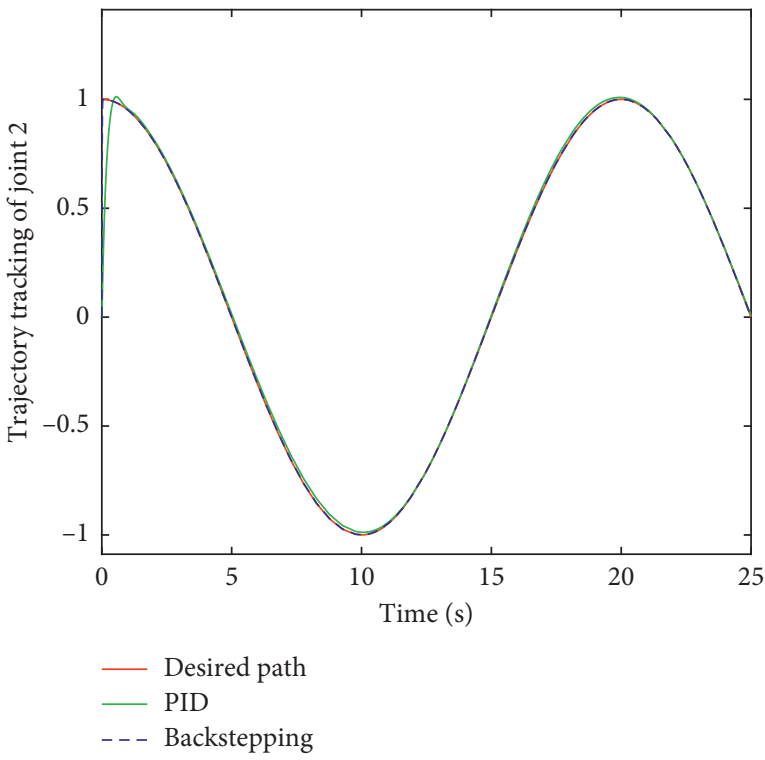

(b)

FIGURE 5: Trajectory tracking of two-link robotic manipulator. (a) $1^{\#}$ link with $q_{1}$. (b) $2^{\#}$ link with $q_{2}$.

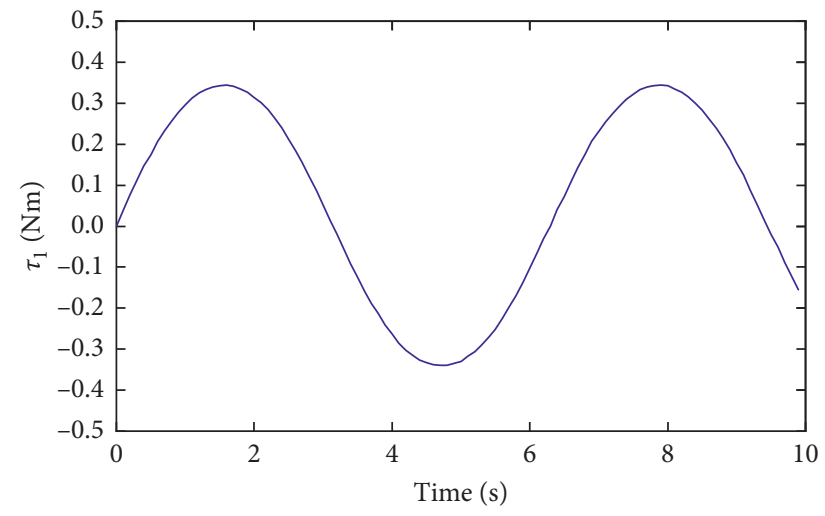

(a)

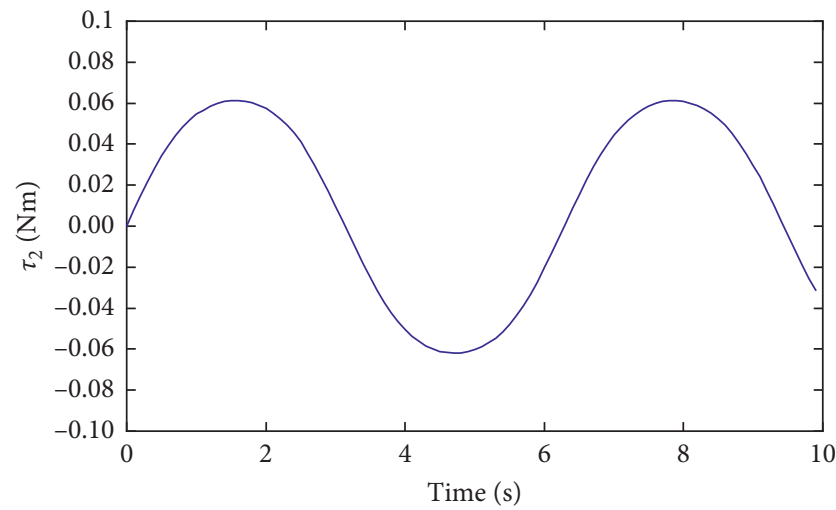

(b)

FIgURE 6: Actuated torque in the joint space as control signal. (a) $\tau_{1}$. (b) $\tau_{2}$. 


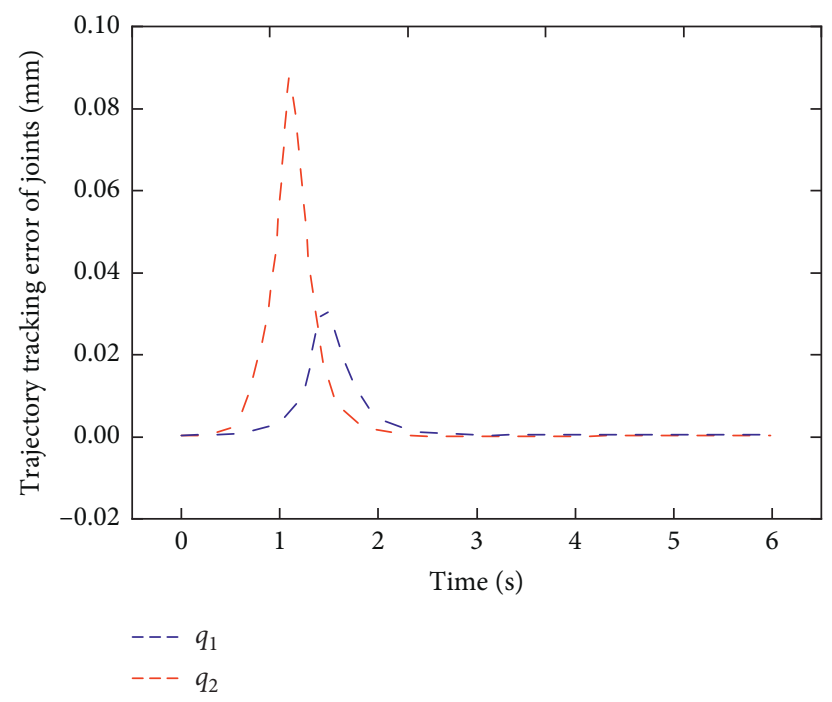

FIGURE 7: Trajectory tracking errors of the two-link robotic manipulator in joint space.

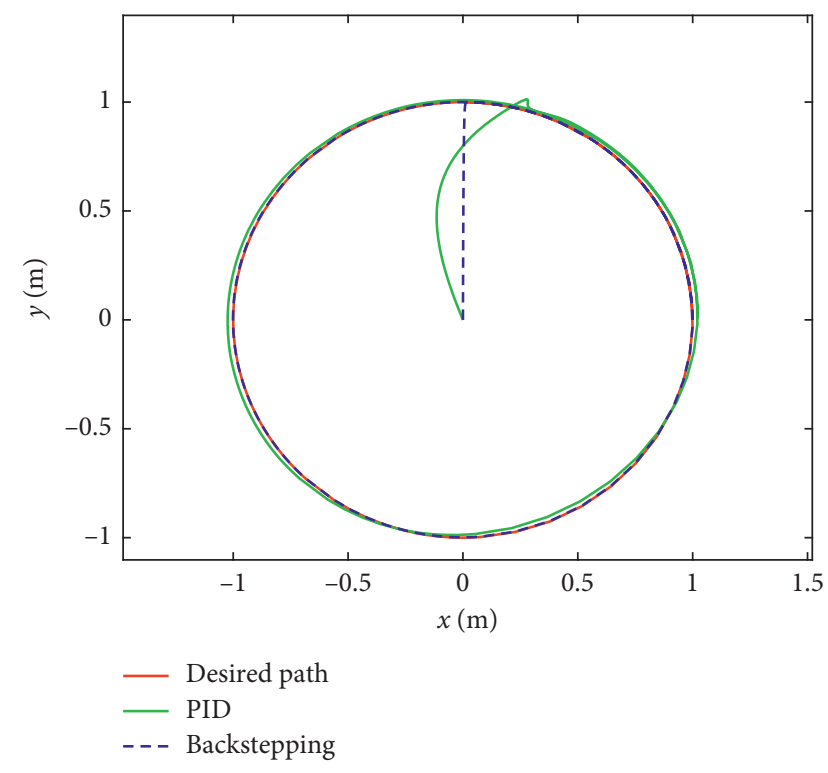

FIGURE 8: Trajectory tracking performance in the operating space of the end-effector.

$$
M=\left[\begin{array}{cc}
k_{1}+\lambda^{T} h \lambda & h \lambda-\frac{1}{2} I \\
h \lambda-\frac{1}{2} I & h
\end{array}\right] .
$$

By giving the proper values of $k_{1}, h$, and $\lambda$, the matrix $M$ can be positive, and $\dot{V}_{3} \leq 0$, and the stability of the robotic manipulator is guaranteed.

\section{Simulation and Discussion}

In this section, a two-link robotic manipulator is utilized to verify the effectiveness of the proposed control strategy. The structural parameters are described in Table 1, and the

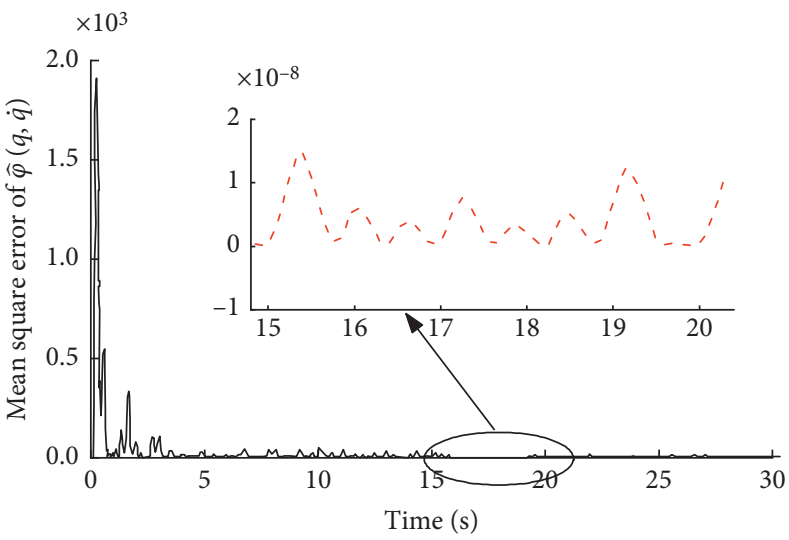

Figure 9: Mean square error of the estimation function $\widehat{\varphi}(q, \dot{q})$.

dynamic equation is given by (35). The desired trajectory tracking of two joints is $\theta_{1 d}=\theta_{2 d}=0.5 \sin (t)$.

The parameter values used in the adaptive backstepping sliding mode control system are $\lambda=\operatorname{diag}[5,5]$, $k_{1}=\operatorname{diag}[50,50], h=\operatorname{diag}[3,3]$, and the external disturbance is added by a pulse signal $\phi=0.5 \delta(t)$ in $6 \mathrm{~s}$, which is used to verify the robustness and stability of the two-link robotic manipulator system. The schematic for backstepping algorithm by using Matlab ${ }^{@}$ software is shown in Figure 3, and the schematic for the proposed control strategy is shown in Figure 4.

Figure 5 shows the trajectory tracking performance of the proposed control with the actuated torque in joint space $\tau_{1}$ and $\tau_{2}$, shown in Figure 6, respectively.

The trajectory tracking errors of the two joints are shown in Figure 7, respectively. The maximum tracking error of the $1^{\#}$ joint is equal to $0.0296 \mathrm{~mm}$ and in $1.6 \mathrm{~s}$ is $5.92 \%$. The maximum tracking error of the $2^{\#}$ joint is equal to $0.0896 \mathrm{~mm}$ and in $1.2 \mathrm{~s}$ is $17.92 \%$ and in $2.2 \mathrm{~s}$ tends to convergence. However, the convergence time of $1^{\#}$ joint is in $2.8 \mathrm{~s}$, which is affected by the coupling of $2^{\#}$ joint. 


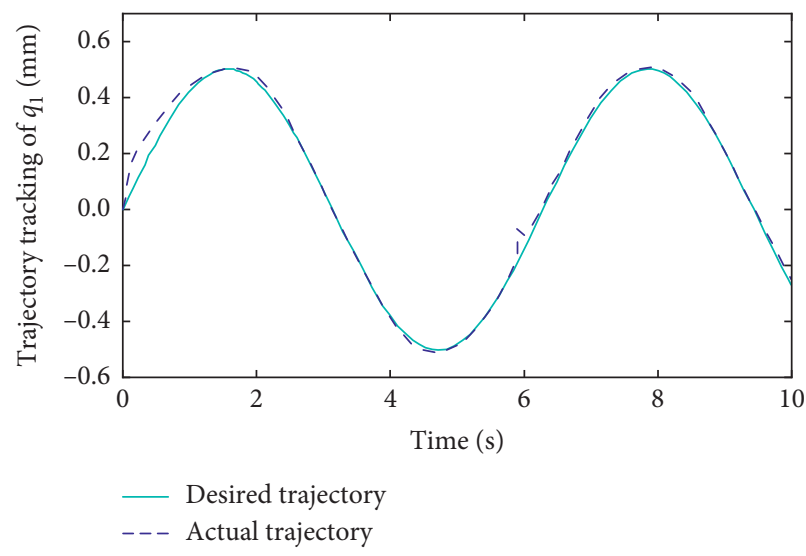

(a)

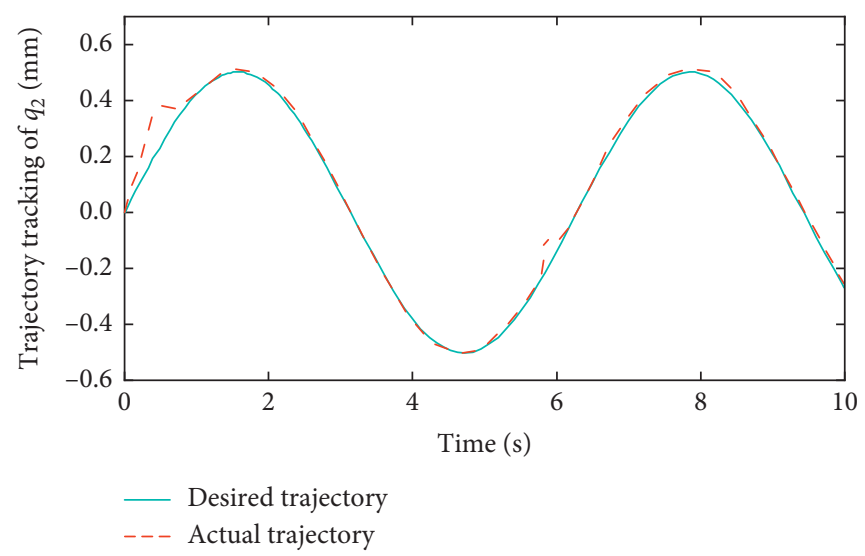

(b)

FIgURE 10: Trajectory tracking of the two-link robotic manipulator under external disturbances.

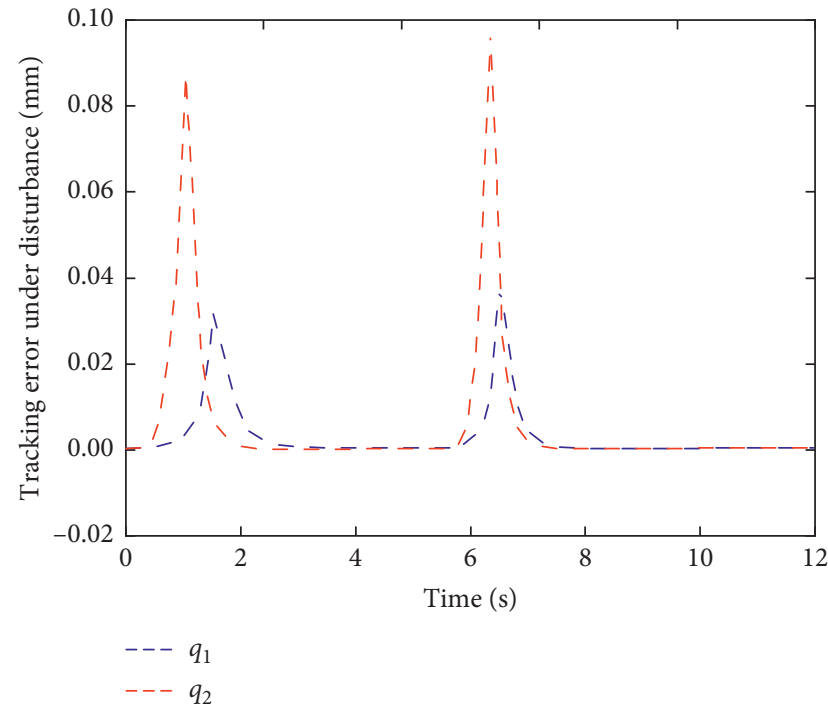

FIgURE 11: Tracking errors of the two-link robotic manipulator under external disturbances.

The trajectory tracking performance in end-effector space (operating space) is shown in Figure 8. Furthermore, the mean square error of the estimation function $\widehat{\varphi}(q, \dot{q})$ is derived, shown in Figure 9. Figure 9 shows that the maximum estimation error is $1.8 \times 10^{-3}$, and the minimum error is $1.68 \times 10^{-8}$, meaning that the proposed controller has a good effect in nonlinear estimation and tracking abilities.

To verify the robustness of the proposed control system, an external disturbance is added to the system in $6 \mathrm{~s}$. The trajectory tracking of the two-link robotic manipulator under external disturbances is shown in Figure 10. Furthermore, the trajectory tracking errors in joint space are shown in Figure 11. It shows that the maximum tracking error of the $1^{\#}$ joint is equal to $0.0316 \mathrm{~mm}$, and the maximum tracking error of the $2^{\#}$ joint is equal to $0.076 \mathrm{~mm}$. According to the given external disturbance, the percentages of the tracking errors are $6.32 \%$ and $15.2 \%$, respectively.

\section{Conclusions}

In this article, an adaptive backstepping sliding mode control subject to external disturbance is proposed. The dynamic model of the robotic manipulator is built by considering the coupling and nonlinear characteristics, and the estimate function of these nonlinear factors is proposed and used for the equivalent control law of sliding mode control. The control system is designed by the backstepping algorithm, and the stability and robustness of the two-link robotic manipulator are analyzed. Simulation results show that the proposed control system has good tracking performance and strong robustness for the external disturbance.

\section{Data Availability}

The data used to support the findings of this study are available from the corresponding author upon request.

\section{Conflicts of Interest}

The authors declare that they have no conflicts of interest.

\section{Acknowledgments}

The authors are grateful for the financial support from the National Natural Science Foundation of China (Grant no. 51165009) and Innovation School Project of Education Department of Guangdong Province, China (Grant nos. 2017KZDXM060 and 2018KCXTD023).

\section{References}

[1] B. Lavinia, P. Glovanni, C. N. Joseph, L. O. Keith, and V. Pietro, "Adaptive dynamic control for magnetically actuated medical robots," IEEE Robotics and Automation Letters, vol. 4, no. 4, pp. 3633-3640, 2019.

[2] H. Wang and Y. Xie, "Adaptive inverse dynamics control of robots with uncertain kinematics and dynamics," Automatica, vol. 45, no. 9, pp. 2114-2119, 2009. 
[3] H. Shi, Y. Liang, and Z. Liu, "An approach to the dynamic modeling and sliding mode control of the constrained robot," Advances in Mechanical Engineering, vol. 9, no. 2, pp. 1-10, 2017.

[4] R. Yang, C. Yang, M. Chen, and A. S. Annamalai, "Discretetime optimal adaptive RBFNN control for robot manipulators with uncertain dynamics," Neurocomputing, vol. 234, pp. 107-115, 2017.

[5] S. Mohammad, N. Pedro, and B. Richard, "Robot dynamics: a recursive algorithm for efficient calculation of Christoffel symbols," Mechanism and Machine Theory, vol. 142, p. 103589, 2019.

[6] J. Wu, J. Wang, and Z. You, "An overview of dynamic parameter identification of robots," Robotics and ComputerIntegrated Manufacturing, vol. 26, pp. 414-419, 2010.

[7] M. Wang, Y. Zhang, and H. Ye, "Dynamic learning from adaptive neural control of uncertain robots with guaranteed full-state tracking precision," Complexity, vol. 2017, Article ID 5860649, 14 pages, 2017.

[8] M. Gautier, A. Janot, and P. O. Vandanjon, "A new closedloop output error method for parameter identification of robot dynamics," IEEE Transactions on Control Systems Technology, vol. 21, no. 3, pp. 428-444, 2013.

[9] X. Yang, X. Zhang, S. Xu, Y. Ding, K. Zhu, and P. X. Liu, “An approach to the dynamics and control of uncertain robot manipulators," Algorithm, vol. 12, no. 66, 2019.

[10] X. Li, X. Wang, and J. Wang, "A kind of Lagrange dynamic simplified modeling method for multi-DOF robot1," Journal of Intelligent \& Fuzzy Systems, vol. 31, no. 4, pp. 2393-2401, 2016.

[11] H. B. Mojtaba, M. Vahid, R. G. Reza, A. N. Seyyed, and E. Arash, "Dynamic analysis, simulation, and control of a 6DOF IRB-120 robot manipulator using sliding mode control and boundary layer method," Journal of Central South University, vol. 25, pp. 2219-2214, 2018.

[12] J. Y. Cheong, S. I. H. Han, and J. M. Lee, “Adaptive fuzzy dynamic surface sliding mode position control for a robot manipulator with friction and deadzone," Mathematical Problems in Engineering, vol. 2013, Article ID 161325, 15 pages, 2013.

[13] S. He, J. Song, and F. Liu, "Robust finite-time bounded controller design of time-delay conic nonlinear systems using sliding mode control strategy," IEEE Transactions on Systems, Man, and Cybernetics: Systems, vol. 48, no. 11, pp. 1863-1873, 2018.

[14] X. Cheng, X. Tu, Y. Zhou, and R. Zhou, “Active disturbance rejection control of multi-joint industrial robots based on dynamic feedforward," Electronics, vol. 8, no. 5, p. 591, 2019.

[15] R.-J. Wai and R. Muthusamy, "Design of fuzzy-neural-network-inherited backstepping control for robot manipulator including actuator dynamics," IEEE Transactions on Fuzzy Systems, vol. 22, no. 4, pp. 709-722, 2014.

[16] S. He, W. Lyu, and F. Liu, "Robust $\mathrm{H}_{\infty}$ sliding mode controller design of a class of time-delayed discrete conic-type nonlinear systems," IEEE Transactions on Systems, Man, and Cybernetics: Systems, 2019.

[17] S.-H. Yen, P.-C. Tang, Y.-C. Lin, and C.-Y. Lin, “A sensorless and low-gain brushless DC motor controller using a simplified dynamic force compensator for robot arm application," Sensors, vol. 19, no. 14, p. 3171, 2019.

[18] B. Deng, J. Wang, and X. Fei, "Synchronizing two coupled chaotic neurons in external electrical stimulation using backstepping control," Chaos Solitons \& Fractals, vol. 29, no. 1, pp. 182-189, 2006.
[19] J. Zhou, C. Wen, and Y. Zhang, "Adaptive backstepping control of a class of uncertain nonlinear systems with unknown backlash-like hysteresis," IEEE Transactions on Automatic Control, vol. 49, no. 10, pp. 1751-1757, 2004.

[20] S. He, H. Fang, M. Zhang, F. Liu, and Z. Ding, "Adaptive optimal control for a class of nonlinear systems: the online policy iteration approach," IEEE Transactions on Neural Networks and Learning Systems, vol. 31, no. 2, pp. 549-558, 2020.

[21] S. He, H. Fang, M. Zhang, F. Liu, X. Luan, and Z. Ding, "Online policy iterative-based $\mathrm{H} \infty$ optimization algorithm for a class of nonlinear systems," Information Sciences, vol. 495, pp. 1-13, 2019.

[22] R. Nie, S. He, F. Liu, and X. Luan, "Sliding mode controller design for conic-type nonlinear semi-markovian jumping systems of time-delayed Chua's circuit," IEEE Transactions on Systems, Man, and Cybernetics: Systems, 2019.

[23] H. Chaudhary, V. Panwar, R. Prasad, and N. Sukavanam, "Adaptive neuro fuzzy based hybrid force/position control for an industrial robot manipulator," Journal of Intelligent Manufacturing, vol. 27, no. 6, pp. 1299-1308, 2016.

[24] C. Yang, Y. Jiang, W. He, J. Na, Z. Li, and B. Xu, "Adaptive parameter estimation and control design for robot manipulators with finite-time convergence," IEEE Transactions on Industrial Electronics, vol. 65, no. 10, pp. 8112-8123, 2018.

[25] T. Y. Vu, Y. N. Wang, and V. C. Pham, "Robust adaptive sliding mode neural networks control for industrial robot manipulators," International Journal of Control, Automation and Systems, vol. 17, no. 3, pp. 783-792, 2019.

[26] A. S. Lafmejani, M. T. Masouleh, and A. Kalhor, "Trajectory tracking control of a pneumatically actuated 6-DOF GoughStewart parallel robot using backstepping-sliding mode control and geometry-based quasi forward kinematic method," Robotics and Computer Integrated Manufacturing, vol. 54, pp. 96-114, 2018.

[27] L. Qin, F. Liu, and L. Liang, "The application of adaptive backstepping sliding mode for hybrid humanoid robot arm trajectory tracking control," Advances in Mechanical Engineering, vol. 6, Article ID 307985, 2014.

[28] I. O. Aksu and R. Coban, "Sliding mode PI control with backstepping approach for MIMO nonlinear cross-coupled tank systems," International Journal of Robust and Nonlinear Control, vol. 29, no. 6, pp. 1854-1871, 2019.

[29] X. Xu, W. Wu, and W. Zhang, "Sliding mode control for a nonlinear aeroelastic system through backstepping," Journal of Aerospace Engineering, vol. 31, no. 1, Article ID 04017080, 2018.

[30] C. Yang, H. Wu, Z. Li, W. He, N. Wang, and C.-Y. Su, "Mind control of a robotic arm with visual fusion technology," IEEE Transactions on Industrial Informatics, vol. 14, no. 9, pp. 3822-3830, 2018.

[31] C. Ren, S. He, X. Luan, F. Liu, and H. R. Karimi, "Finite-time $\mathrm{L}_{2}$-gain asynchronous control for continuous-time positive hidden markov jump systems via T-S fuzzy model approach," IEEE Transactions on Cybernetics, 2020.

[32] I. Bonilla, F. Reyes, M. Mendoza, and E. J. González-Galván, "A dynamic-compensation approach to impedance control of robot manipulators," Journal of Intelligent \& Robotic Systems, vol. 63, no. 1, pp. 51-73, 2011.

[33] C. Yang, C. Chen, W. He, R. Cui, and Z. Li, "Robot learning system based on adaptive neural control and dynamic movement primitives," IEEE Transactions on Neural Networks and Learning Systems, vol. 30, no. 3, pp. 777-787, 2019. 